\title{
Quiste gigante de la túnica albugínea
}

\section{Giant cyst of the tunica albuginea}

\author{
Cesar Iván Peña-Ruelas,,$^{1,2}$ María del Mar Rentería-Manzanilla, ${ }^{1}$ Elsa Edith de la Rosa-Vélez ${ }^{2}$
}

\begin{abstract}
Resumen
ANTECEDENTES: Los quistes de la túnica albugínea son tumores benignos, con incidencia de 0.3 a $0.76 \%$. La mayoría de los casos son intratesticulares y el tamaño promedio es de 2 a $5 \mathrm{~mm}$. Cuando los quistes son gigantes, según su localización y tamaño, pueden malinterpretarse como tumores malignos.

CASO CLÍNICO: Paciente masculino de 55 años, con aumento progresivo en la región testicular izquierda, de 30 años de evolución. El ultrasonido y la tomografía computada mostraron un tumor quístico, heterogéneo, de contorno definido que comprimía el testículo izquierdo, por lo que se realizó orquiectomía. El estudio anatomopatológico confirmó la coexistencia del quiste, de $20 \times 16 \mathrm{~cm}$, uniloculado, con contenido líquido, localizado en la túnica albugínea; microscópicamente tenía recubrimiento epitelial simple, de tipo plano o cúbico sin atipia. El diagnóstico final fue: quiste de túnica albugínea.
\end{abstract}

CONCLUSIONES: El tratamiento de los quistes de la túnica albugínea varía en función del tamaño y síntomas del paciente. El caso aquí reportado representa el quiste de túnica albugínea de mayor tamaño informado hasta la fecha.

PALABRAS CLAVE: Quiste intratesticular; túnica albugínea.

\section{Abstract}

BACKGROUND: Cysts of the tunica albuginea are benign tumors with an incidence that varies from 0.3 to $0.76 \%$. The majority of cases are classified as intratesticular and average size is 2 to $5 \mathrm{~mm}$. When the cysts are very large, due to location and size, they can be misinterpreted as malignant.

CLINICAL CASE: A 55-year-old man presented with progressive enlargement of the left testicular region of 30-year progression. Ultrasound and computed tomography studies identified a well-defined, heterogeneous cystic tumor that compressed the left testis, for which orchiectomy was performed. The anatomopathologic study confirmed a 20 $\times 16 \mathrm{~cm}$, unilocular cyst with fluid content, located in the tunica albuginea. Microscopically, it was lined with a flat or cuboidal epithelium with no atypia. Diagnosis was a cyst of the tunica albuginea.

CONCLUSION: Treatment of cysts of the tunica albuginea may vary according to size and symptomatology. The case presented herein is the largest cyst of the tunica albuginea to yet be reported.

KEYWORDS: Intratesticular cyst; Tunica albuginea.
${ }^{1}$ Departamento de Anatomía Patológica, UMAE Hospital de Especialidades 25 Instituto Mexicano del Seguro Social, Monterrey, Nuevo León.

${ }^{2}$ Laboratorio DIPAC. Monterrey, México.

Recibido: febrero 2018

Aceptado: agosto 2018

Correspondencia Cesar Iván Peña Ruelas patologia.cipr@gmail.com

Este artículo debe citarse como Peña-Ruelas $\mathrm{Cl}$, Rentería-Manzanilla MM, de la Rosa-Vélez EE. Quiste gigante de la túnica albugínea. Rev Mex Urol. 2018 sept-oct;78(5):385-388. DOI: https://doi.org/10.24245/revmexurol.v78i5.2011 


\section{ANTECEDENTES}

Los quistes de la túnica albugínea son tumores testiculares benignos poco frecuentes, cuya incidencia varía de 0.3 a $0.76 \% .^{1-2}$ El primer reporte fue efectuado en 1929 por Frater, quien sugirió el posible origen traumático. ${ }^{3}$ La mayoría de los casos manifiesta quistes de 2 a $5 \mathrm{~mm}^{4}{ }^{4}$

\section{CASO CLÍNICO}

Paciente masculino de 55 años, sin antecedentes urogenitales de traumatismo ni intervenciones quirúrgicas. Refirió iniciar su padecimiento hace 30 años, con aumento progresivo en la región testicular izquierda. A la exploración física se identificó una tumoración en la bolsa escrotal izquierda a expensas de su contenido, no dolorosa a la palpación. La determinación de marcadores tumorales se encontró dentro de los parámetros normales: antígeno carcinoembrionario $2.37 \mathrm{ng} / \mathrm{mL}$, $\alpha$-fetoproteína $7.27 \mathrm{ng} / \mathrm{mL}$ y hCG- $\beta 1.20 \mathrm{mUl} / \mathrm{mL}$.

El ultrasonido (Figura 1A) mostró, en la región posterolateral del testículo izquierdo, una imagen alargada, ovalada, con interior de aspecto heterogéneo, además de múltiples imágenes circulares anecoicas de diferentes tamaños, rodeada por corteza anormal de parénquima ecogénico. Los cortes axial y coronal de la tomografía computada (Figuras 1B y 1C) evidenciaron una imagen ovalada, con interior heterogéneo de predominio hipodenso y rodeada por corteza parenquimatosa, ligeramente hiperdensa, en relación con los tejidos blandos; el testículo contralateral se observó desplazado, pero sin alteración en el parénquima. No se identificaron adenopatías.

Por el tamaño de la lesión y los estudios de gabinete no concluyentes se llevó a cabo la orquiectomía radical izquierda. El servicio de Anatomía Patológica reportó un espécimen de $20 \times 16 \mathrm{~cm}$, con superficie marrón-clara, opaca y renitente; al corte se observó quístico, con abundante salida de líquido color marrón-claro, con grumos blanquecinos; la pared midió 0.3$0.5 \mathrm{~cm}$ de grosor; la superficie interna mostraba coloración marrón con zonas de hemorragia (Figura 2). El cordón espermático no mostró alteraciones.

Los cortes histológicos, teñidos con hematoxilina-eosina (Figura 3), objetivaron una lesión quística, conformada por una pared fibrosa recubierta por epitelio simple, de tipo plano o cúbico sin atipia, con áreas extensas esfacela-
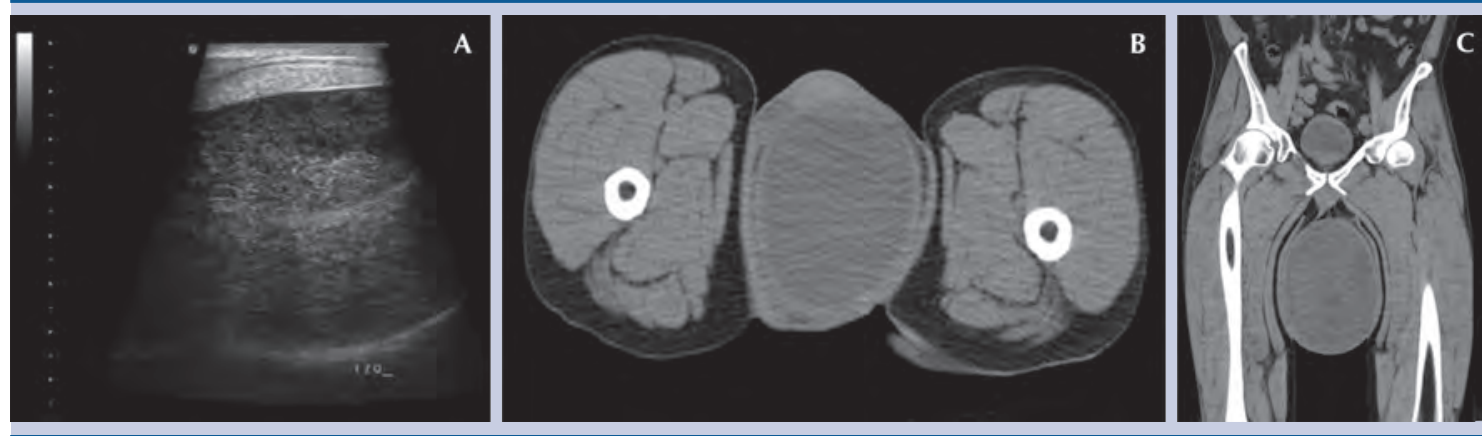

Figura 1. A) Ultrasonido que muestra una estructura quística de interior heterogéneo. B y C) Tomografía computada en corte axial y coronal que muestra un quiste de interior heterogéneo, de contorno bien definido y que desplaza el testículo contralateral. 


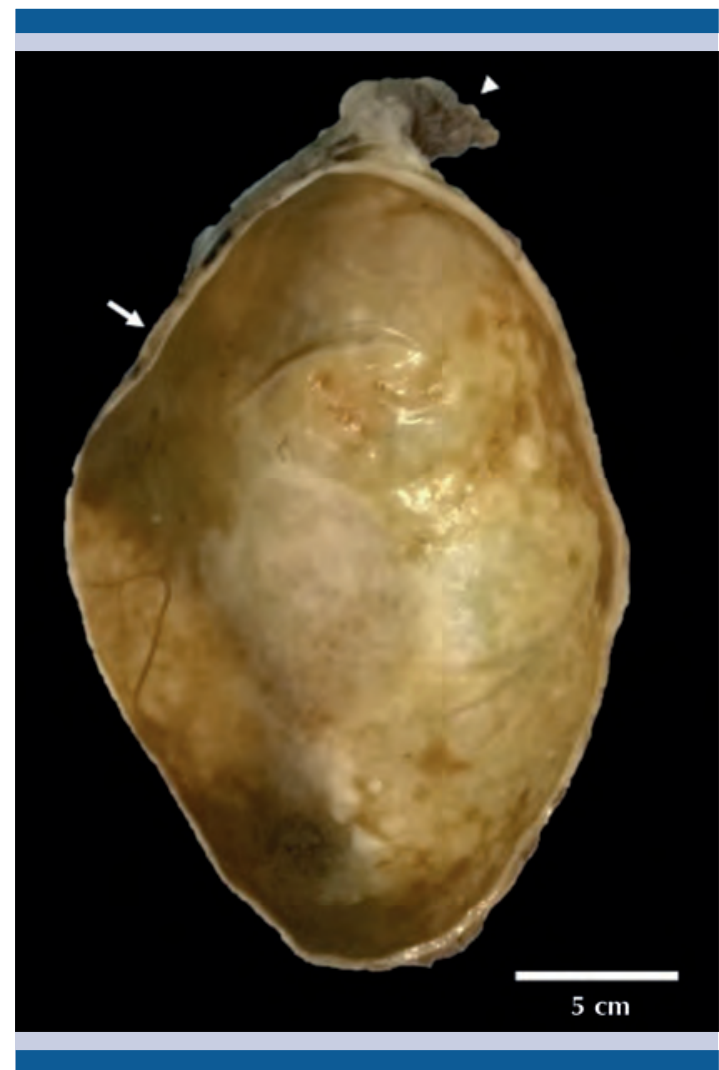

Figura 2. Quiste unilocular con zonas de hemorragia, escaso remanente de parénquima testicular (flecha) y cordón espermático congestivo (punta de flecha).

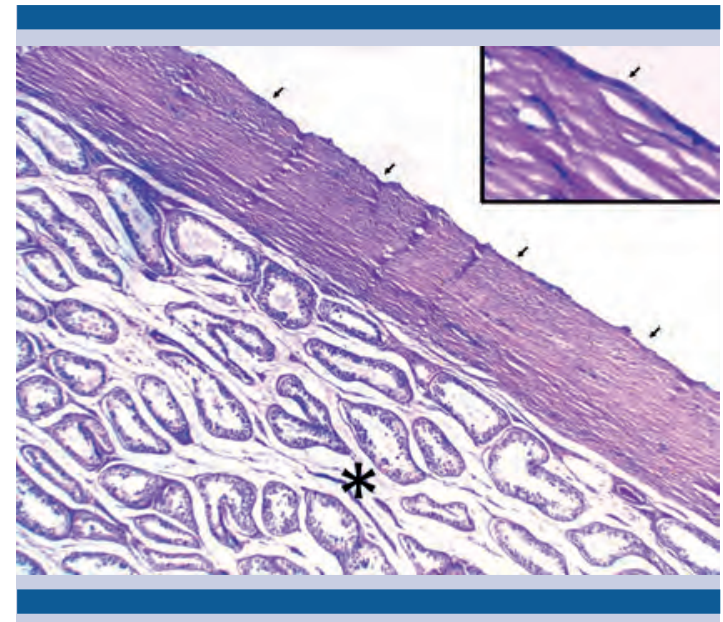

Figura 3. Quiste de pared fibrosa recubierto por epitelio simple (flechas), adyacente al parénquima testicular, con atrofia y edema (asterisco). das; el parénquima testicular mostró atrofia del componente germinal y edema del intersticio. El diagnóstico establecido fue: quiste de la túnica albugínea.

\section{DISCUSIÓN}

Los quistes de la túnica albugínea son nódulos palpables que se expresan entre la quinta y sexta décadas de la vida. ${ }^{2}$ La patogénesis es incierta; sin embargo, se ha sugerido su procedencia de restos mesoteliales o de los ductos eferentes que pasan a través de la túnica albugínea. ${ }^{3,4}$

Otras teorías consideran un origen multifactorial, como traumatismos, isquemia o procesos inflamatorios, no coincidentes con el caso reportado en este estudio. ${ }^{5}$ Martínez-Berganza y su grupo reportaron que los traumatismos pueden provocar la formación del quiste; sin embargo, en algunos pacientes no se ha identificado esta relación, pues olvidaron porqué sucedió el evento, o quizá fueron provocados por microtraumatismos. $^{2}$

La mayor parte de los estudios clasifica los quistes de la túnica albugínea como intratesticulares debido a su relación con el parénquima testicular; sin embargo, algunos pacientes han reportado crecimiento en la superficie externa de la túnica albugínea. ${ }^{6}$ Son fácilmente palpables, incluso si son pequeños; cuando alcanzan dimensiones mayores pueden causar dolor $y$, en ocasiones, malinterpretarse como tumores. ${ }^{7}$

El diagnóstico de quiste de la túnica albugínea se establece mediante ecografía, con la intención de valorar si corresponde a una masa intra o extratesticular, dato relevante porque la mayor parte de las masas extratesticulares son benignas, pero las intratesticulares tienen gran probabilidad de evolucionar a malignidad. ${ }^{8}$ El quiste de la túnica albugínea tiene características ultrasonográficas variables: de un quiste simple, unilocular 
o multiloculado, a quistes de apariencia compleja, con material ecogénico correspondiente a detritus inflamatorios y calcificación. ${ }^{4}$

Los quistes pequeños y asintomáticos pueden tratarse conservadoramente, con escisión de la masa y preservación del parénquima testicular. Los quistes grandes que desplazan el parénquima testicular pueden tratarse con orquiectomía, como sucedió en nuestro paciente. ${ }^{3}$

El caso aquí reportado expresó un quiste intratesticular heterogéneo, por lo que inicialmente se sospechó de alguna neoplasia maligna. Sin embargo, la evolución lenta y progresiva coincidía con benignidad de la lesión. Para descartar el proceso maligno se llevó a cabo la determinación de marcadores tumorales y estudios de gabinete que sugirieron una lesión benigna. Debido al gran tamaño de la lesión y a la extensa compresión del parénquima testicular se optó por la orquiectomía. Actualmente el paciente se encuentra sin complicaciones secundarias a la intervención quirúrgica.

La etiología del quiste permanece desconocida, incluso se ha relacionado con isquemia, traumatismos e infección. El único antecedente de relevancia en nuestro paciente fue la hipertensión arterial sistémica descontrolada; sin embargo, las estructuras vasculares observadas en el testículo no mostraron alteraciones, por lo que no podemos establecer una relación entre su origen vascular o isquémico. En otros casos reportados se menciona la asociación de quistes de la túnica albugínea con microtraumatismos continuos, imperceptibles por el paciente, y por ello no se señalan en el interrogatorio de rutina. De acuerdo con la revisión bibliográfica, el caso aquí reportado representa el quiste de la túnica albugínea de mayor tamaño informado hasta la fecha.

\section{REFERENCIAS}

1. Chou SJ, et al. Cyst of the tunica albuginea. Arch Androl 2004;50:89-92.

2. Martínez-Berganza MT, et al. Cyst of the tunica albuginea: sonographic appearance. AJR AM J Roentgenol 1998;170:183-185. DOI: 10.2214/ajr.170.1.9423628

3. Tanaka K, et al. Benign cystic lesion of the tunica albuginea: a case report. Hinyokika Kiyo Urol 2004;50:45-48.

4. Alvarez DM, et al. Sonographic spectrum of the tunica albuginea cyst. J Clin Imaging Sci 2011;1:1. DOI: 10.4103/21567514.73503

5. Rodríguez-Patrón, R, et al. Ecografía testicular. Arch Esp Urol 2006;59(4):441-454. URL: <http://scielo.isciii.es/scielo. php?script=sci_arttext\&pid=S0004-06142006000400013>.

6. Aminu $\mathrm{S}$, et al. Large tunica albuginea cyst mimicking a large hydrocele with a unique ultrasound feature. Cent European J Urol 2011;64(3):184-185. DOI: 10.5173/ ceju.2011.03.art21

7. Valentino $\mathrm{M}$, et al. Cystic lesions and scrotal fluid collections in adults: ultrasound findings. J Ultrasound 2011;14:208215. DOI: 10.1016/j.jus.2011.10.008

8. Velasco-Ruiz M, et al. Evaluación ecográfica de la patología testicular más frecuente. EuroEco 2010;1(3):49-54. URL: < http://www.euroeco.org/vol1_num3_septb2010/ pdf/49_54.pdf $>$. 